\title{
Research on Competitive Intelligence warning model for enterprise technology innovation risk
}

\author{
YANG bo ${ }^{1,}$,, LU Jia-qi ${ }^{2, b}$ \\ ${ }^{1}$ School of Information Management, Jiang Xi University of Finance \& Economics, Nan Chang \\ 330013, China \\ ${ }^{2}$ School of Information Management, Jiang Xi University of Finance \& Economics, Nan Chang \\ 330013, China \\ Ayb_Ih@163.com, blujiaqi20@163.com
}

\begin{abstract}
Keywords:competitive intelligence; early warning;technological innovation risk; modeling
Abstract. This paper analyzes the cause and effect relationship and evolution characteristics of enterprise technology innovation risk competitive intelligence early warning, and constructs the dynamic model of enterprise technology innovation risk competitive intelligence warning. Then the model is simulated on Vensim PLE.The analysis for effectiveness and sensitivity of simulation model indicates the model can be used as the fitting of enterprise technological innovation risk competitive intelligence warning well and reveals the feature and mechanism of enterprise technological innovation risk. The simulation results indicates that timely enterprise technology innovation risk regulation can effectively avoid enterprise performance loss.
\end{abstract}

\section{Introduction}

Enterprise technological innovation is an important driving force for the sustainable development of social economy in the whole country.However, it is a high-risk activity[1].Therefore, how to prevent and control technological innovation risk has become one of the important issues that managers pay attention to.Competitive Intelligence(CI), is the information research on the competitive environment, competitors and competitive strategy[2]. One of the most important functions of CI is to provide the enterprise crisis early warning, which enable enterprises to avoid a "surprise attack".Making full use of CI early warning is an effective way to prevent or reduce enterprise technological innovation risk.CI early warning is able to monitor the trend of technological innovation risk and identify the weak signal before the arrival of the risk in advance, and form early warning plan.In the aspect of enterprise technological innovation risk and its application research on CI early warning model,related research were done in $[3,4,5,6,7]$. At present, the research is mainly focused on the static characteristics and empirical analysis of the influencing factors, early warning indicators and so on,but the research on the construction of risk early warning system model, as well as the technology innovation risk CI early warning process dynamic evolution and the operation mechanism is still insufficient.And our research lays a foundation for further research on the mechanism of enterprise technological innovation risk mechanism.

\section{Technology innovation risk evolution causality system of dynamics modeling}

Enterprise technology innovation risk CI early warning process is actually the process of early warning cycle and feedback of CI risk, which includes the determination of early warning targets, the collection of CI, the analysis of CI and risk prediction, diagnosis and evaluation of risk early warning, reporting and feedback of CI early warning. At the same time, it is also a long-term and periodic dynamic early warning system which is composed of a variety of CI elements. It accords with the characteristics of system dynamics modeling and simulation. The causal relationship of enterprise technology innovation risk competitive intelligence early warning is shown in Fig. 1. 


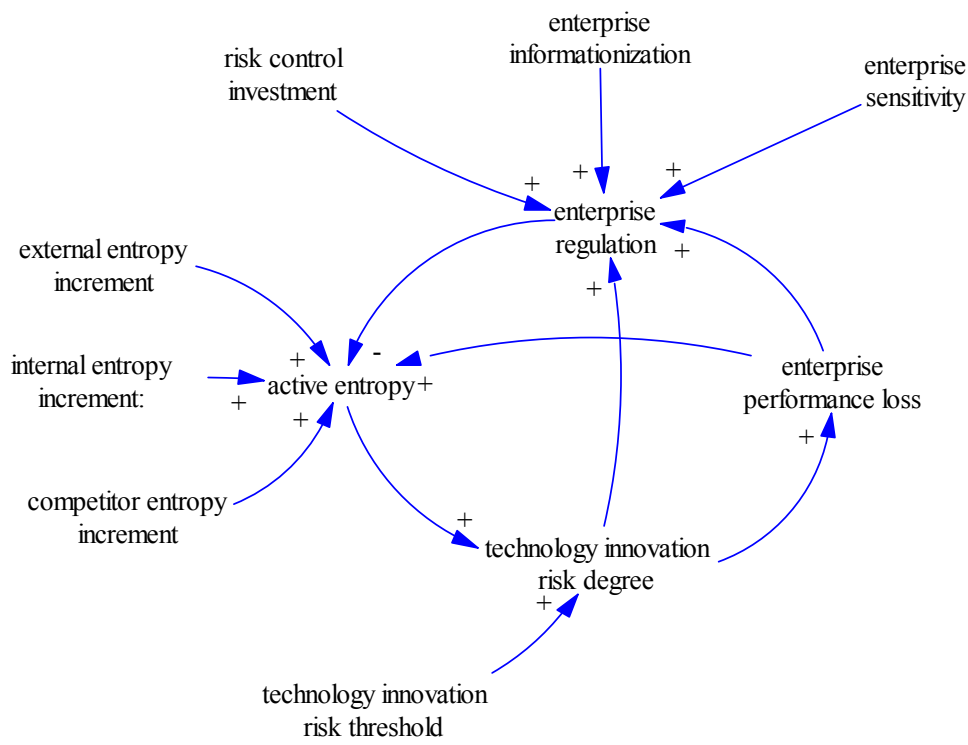

Fig.1. Enterprise technology innovation risk competitive intelligence early warning causality diagram

\section{Enterprise technology innovation risk competitive intelligence early warning model construction}

Model assumptions and system flow diagram. The basic assumptions of the model are:(1)The crisis of technology innovation risk performances as the loss of enterprise performance. (2)The risk threshold of technological innovation is a constant.Enterprise technology innovation risk competitive intelligence warning system flow chart is shown in Fig. 2.

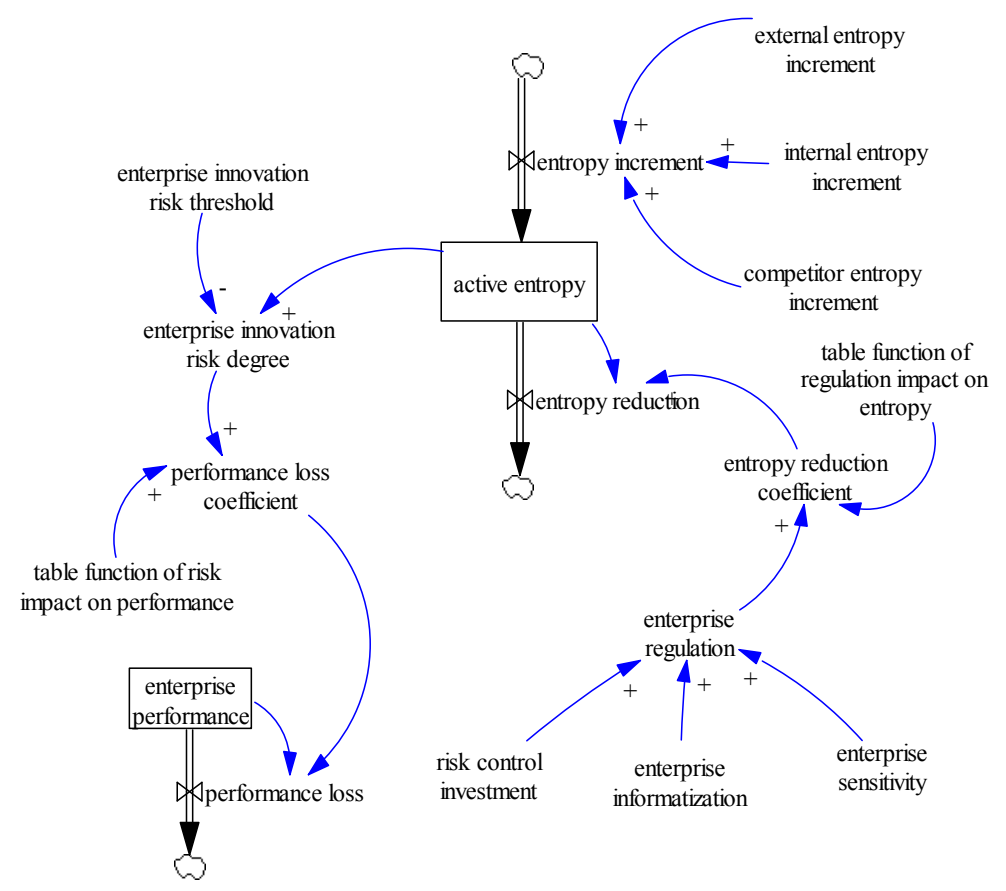

Fig. 2. enterprise technology innovation risk competitive intelligence warning system flow chart

Equation design and description. The main variables of the design equations are described below:

External entropy increment(E-increment) refers to enterprise external environment change.Internal entropy increment(I-increment) refers to unreasonable allocation of internal factors in the process of production and operation of enterprises.Competitor entropy increment( $\mathrm{C}$-increment) 
refers to enterprise competition actions. They all increase enterprise risk.Active entropy characterize state of enterprise risk.Enterprise performance represents the profit level of the enterprise.Risk threshold refers to enterprise risk bearing capacity.

Main functions of the model are as follows:

Entropy increment $=0.3 * \mathrm{C}$-increment $+0.3 *$ E-increment $+0.4 * \mathrm{C}$-increment.

Active entropy=INTEG (Entropy increment -entropy reduction,0).

Table function of regulation impact on entropy

([(0,0)-(100,1)],(0,0),(7.2,0.13),(15,0.27),(30,0.5),(40,0.62),(50,0.72),(62,0.82),(75,0.9),(90,0.97), $(100,1))$.

Degree of risk crisis $=$ IF THEN ELSE(Active entropy-Risk threshold $>0$, (Active entropy-Risk threshold ) $\wedge 0.5,0)$

Performance loss coefficient $=$ table function of risk impact on performance (risk degree)

Enterprise performance $=$ INTEG (-Performance loss, 10000)

Table function of risk impact on performance

([(0,0)-(28,1)],(0,0),(4,0.04),(7.5,0.1),(11,0.16),(15,0.25),(17,0.32),(19,0.385),(22,0.5),(26,0.7),(3 $0,1))$

\section{Enterprise technology innovation risk evolution simulation and analysis}

The model is constructed and simulated on VEMSIM PLE platform. Set the simulation time for 100 months, the stock of active entropy initial value is set to 0 , the initial value of the stock of enterprise performance is set to 10000 .

The basic operation of system when the feedback loop is closed. When enterprise is in the condition of continuous entropy, and enterprise regulation feedback loop is closed, technological innovation risk evolution was identified by simulation. The parameters of the system are set as shown in Table 1.The results of the model simulation run is shown in Fig. 3.,Fig. 4., Fig. 5, Fig. 6. and Fig. 7.

Table 1. parameter values of the system when the feedback is closed

\begin{tabular}{|c|c|c|c|c|c|c|c|}
\hline parameter & E-increment & I-increment & C-increment & threshold & investment & informationization & sensitivity \\
\hline value & 30 & 30 & 20 & 2000 & 0 & 0 & 0 \\
\hline
\end{tabular}

In Fig.3, entropy increment keeps at 27.In Fig.4,active entropy increases uniformly.In Fig.5 degree of risk keeps at 0 before 75 months and increases from fast to slow 75 months later.In Fig.6, influence coefficient curve of performance loss is similar to the change of degree of risk, since they are positively correlated.In Fig.7,enterprise performance remains unchanged before 75 months,but declines rapidly to 0 after 75 months, The curve change can be explained that:before 75 months, active entropy increases continuously, but doesn't exceed risk threshold value.Enterprise performance loss does not occur.However, 75 months later, active entropy exceeds threshold of 2000, enterprise performance reduce, enterprise crisis occurs. 


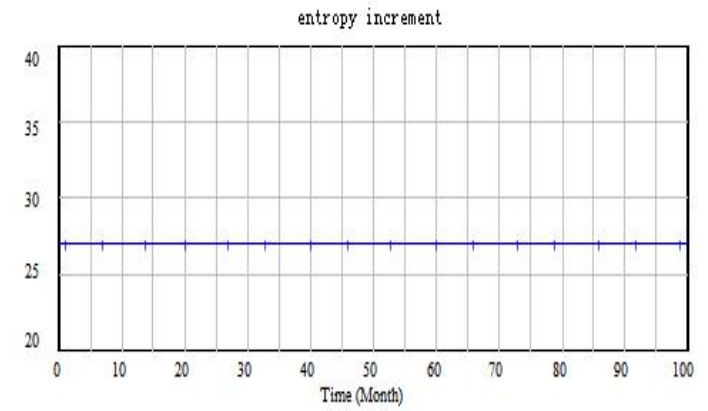

entropy increment: Cusent

Fig. 3. entropy increment

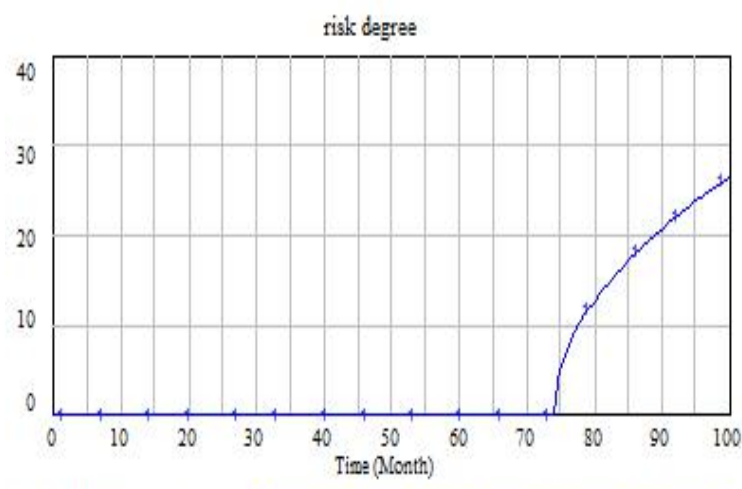

risk degree : Cunent

Fig. 5. risk degree

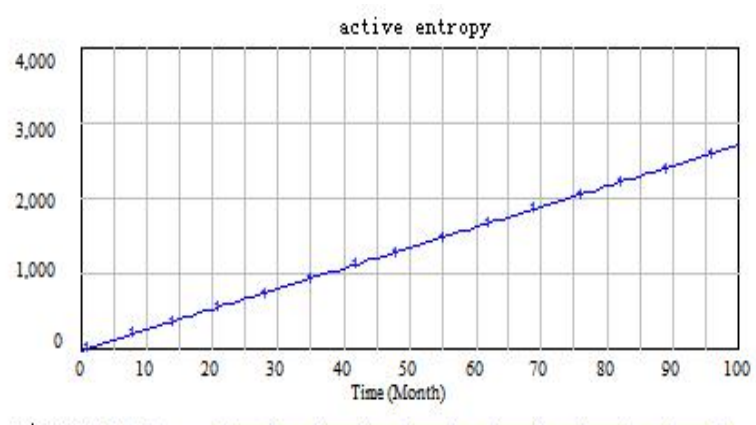

Fig. 4. active entropy

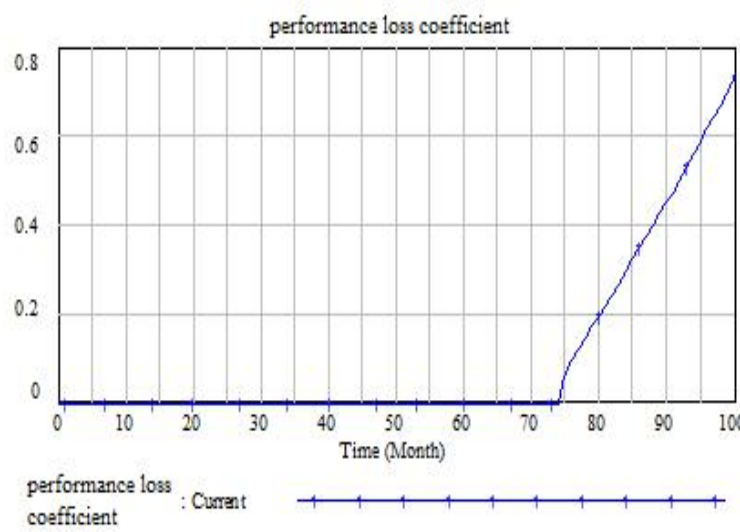

Fig. 6. performance loss coefficient

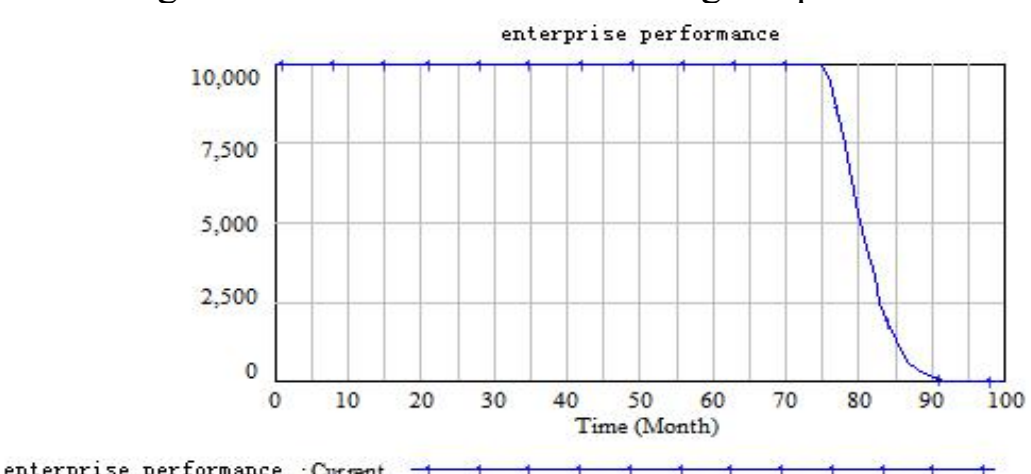

Fig. 7.enterprise performance

The basic operation of the system when the feedback loop is open Keep entropy increase and assign enterprise risk sensitivity value.The parameters are set in Table 2. Results of model simulation are shown in Fig. 8., Fig. 19., Fig. 10. and Fig. 11..

Table 2 parameter values of the system when the feedback is turned on

\begin{tabular}{|c|c|c|c|c|c|c|c|}
\hline parameter & E-increment & I-increment & C-increment & threshold & investment & informationization & sensitivity \\
\hline value & 30 & 30 & 20 & 2000 & 10 & 10 & 10 \\
\hline
\end{tabular}

Fig. 8. shows that active entropy curve rises before 60 months and remains unchanged after 60 months. Because the enterprise regulation gradually strengthen, active entropy remain stable before enterprise take risk control measures.In Fig. 9, the degree of risk remains at 0.In Fig. 10,performance loss effect coefficient remains at 0.In Fig.11,enterprise performance remains unchanged.Because active entropy doesn't exceed risk threshold,enterprise doesn't occur crisis. 


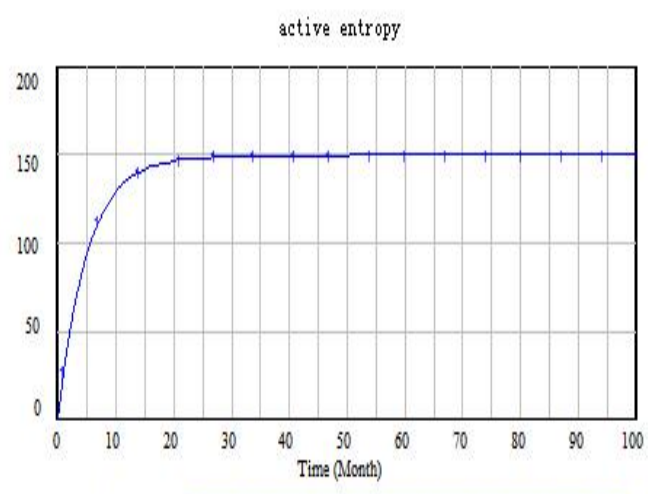

active entropy : Curent

Fig. 8.active entropy

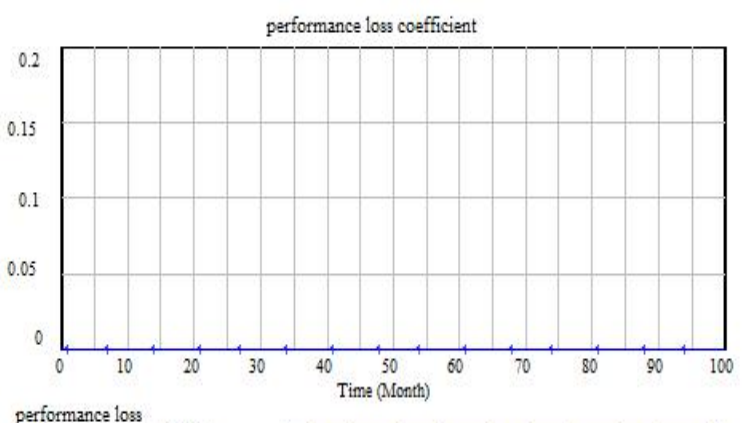
coefficient : Current

Fig. 10 performance loss coefficient

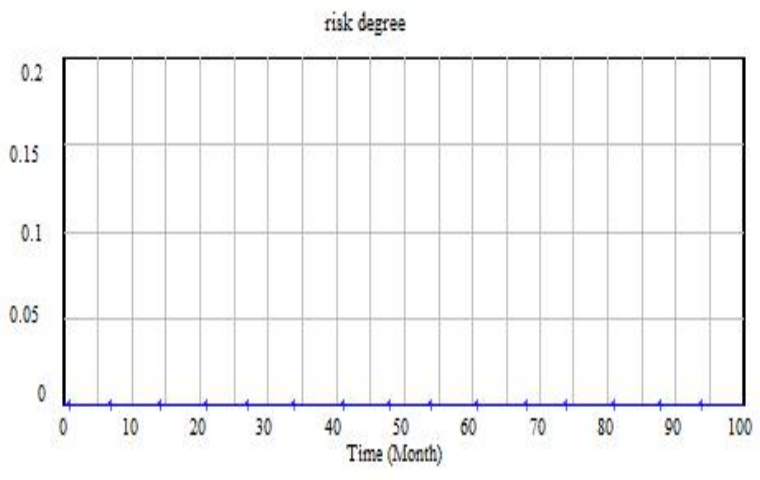

risk degree : Current

Fig. 9. risk degree

enterprise performance

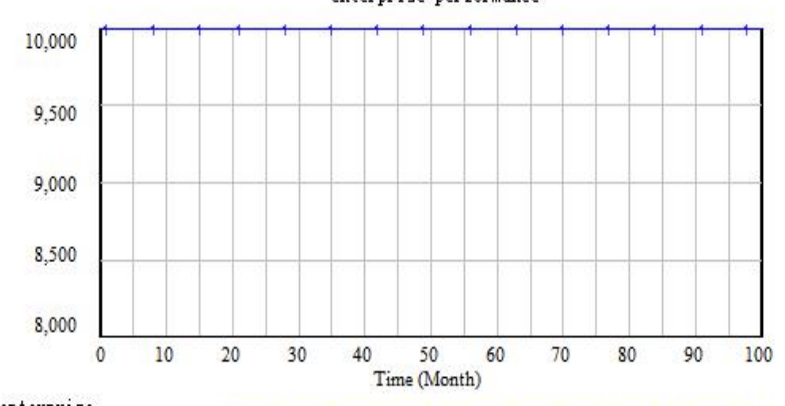

$\underset{\text { performance }}{\text { enterprent }}$

Fig. 11.enterprise performance

Multiple scheme operation The parameters of the system are set as shown in Table 3.The running results are shown in Fig. 12. and Fig. 13.

Table 3 System parameter value adjustment

\begin{tabular}{|l|l|l|l|l|l|l|l|}
\hline Type & E-increment & I-increment & I-increment & threshold & investment & informationization & sensitivity \\
\hline Current & 30 & 30 & 20 & 2000 & 10 & 10 & 10 \\
\hline Current 1 & 30 & 30 & 20 & 2000 & 6 & 10 & 10 \\
\hline Current2 & 30 & 30 & 20 & 2000 & 21 & 10 & 10 \\
\hline
\end{tabular}

When keeping other variables unchanged,Fig. 12.shows that enterprise control is positively related to the risk investment Fig. 13. shows that active entropy is negatively related to risk investment.Because active entropy is under control,the increase rate tends to be reduced if enterprise improves risk control investment.Similarly,active entropy is negatively related to enterprise sensitivity and informatization, as shown in Fig. 14. and Fig. 15.

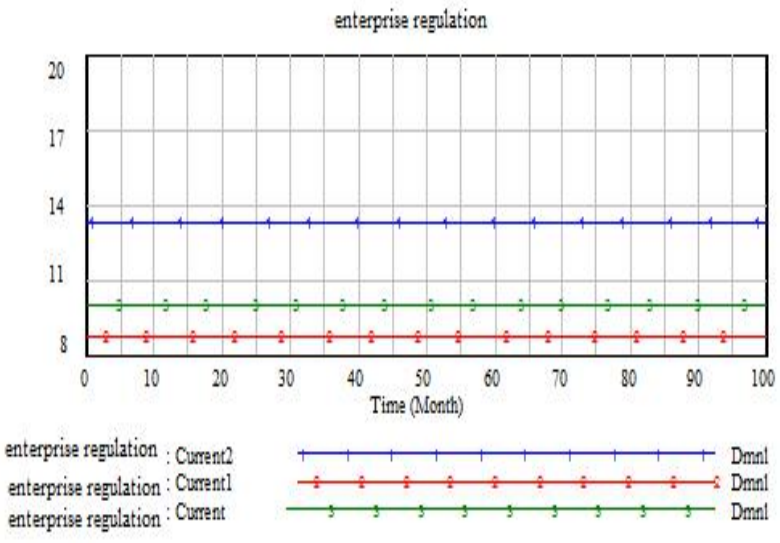

Fig. 12. enterprise regulation

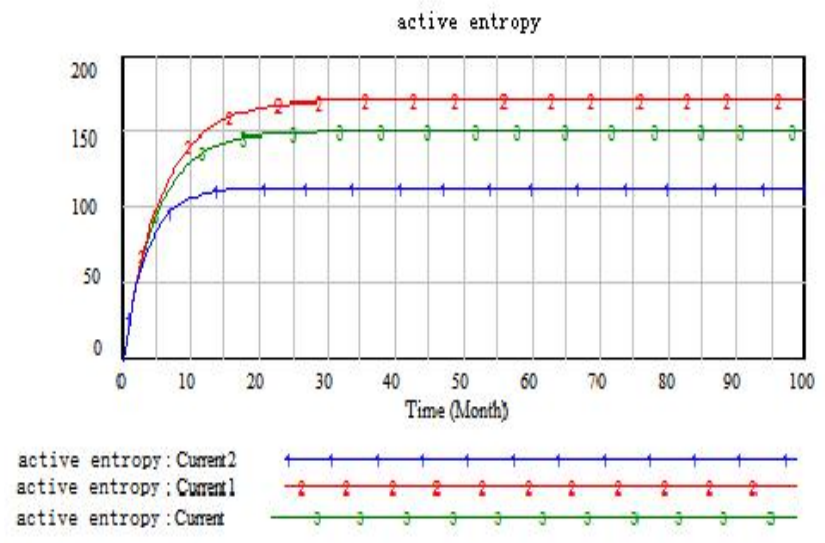

Fig. 13. Active entropy with investment 


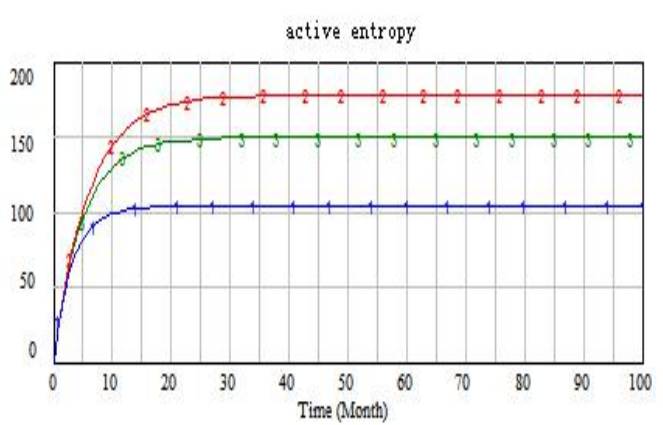

active entropy : Cumert 4 active entrops: Cumert 3

tive entrops : Current

Fig. 14.active entropy with sensitivity

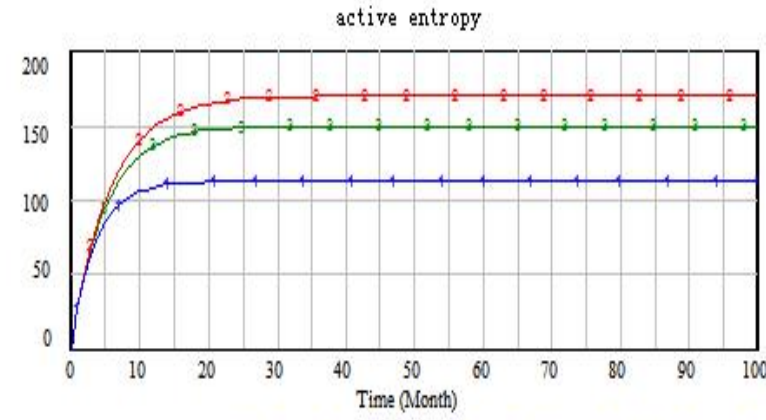

active entropy : Curent6
active entropy : Coment5 active entropy: Cument

Fig. 15. active entropy with informatization

\section{Conclusions}

The simulation analysis of the model indicates that timely enterprise technology innovation risk control measures can effectively avoid enterprise crisis.Active entropy is negatively related with enterprise sensitivity risk control investment and informatization. Therefore, in order to reduce the risk, enterprise should establish a sense of crisis, improve the risk sensitivity of enterprise technology innovation and integrate competitive intelligence early warning process, improve the efficiency of technology innovation risk early warning.

However, real enterprise technology innovation risk competitive intelligence warning is a very complicated and abstract evolution process, this model has some limitations, such as :The influence factors in the model is considered not fully comprehensive and settings are simplified.In the further research,Empirical methods will be used to validate the simulation results.

\section{Acknowledgements}

This work was financially supported by the Jiangxi Province Social Science "Twelfth Five Year Plan" project( 15TQ04) and 2016 university research innovation projects of special funds .

\section{References}

[1] Xiaofeng Li, Jiuping Xu: submitted to Journal of Economic System Reform(2008),In Chinese.

[2] Changhuo Bao, Xinzhou Xie, Yan Zhang and $\mathrm{Na} \mathrm{Li:} \mathrm{submitted} \mathrm{to} \mathrm{Journal} \mathrm{of} \mathrm{Chinese} \mathrm{Information}$ Bulletin(2001),In Chinese.

[3] E.Prescott:Competitive Technical Intelligence: A Guide to Design, Analysis, and Action (Oxford University Press, 1997).

[4] C.Desouza:Tech Mining:Exploiting New Technologies for Competitive Advantage(Oxford University Press, 2010).

[5] Yan Li, Xiuling Wang,Yuan Zhang and Xiaowen Liu:submitted to Journal of Information Theory and Practice (2012),In Chinese.

[6] Zhonglu Ceng and Dongmei Zhang: submitted to Journal of Information Theory and Practice (2013),In Chinese.

[7] Keping Wang, Xiaona Feng, Xiao Zhang,Xinyan Liu: submitted to Journal of Information Science (2014),In Chinese. 